\title{
Evaluation of Short-Distance Airborne Infection Risk Using a Cough Generator
}

\author{
Wei Ling $^{1 *}$, Maho Ichikawa ${ }^{2 *}$, Kaho Hashimoto ${ }^{1}$, Masayuki Ogata ${ }^{1}$, Hitomi Tsutsumi ${ }^{*}$, Shoichi Morimoto ${ }^{4 *}$ Shin-ichi \\ Tanabe $^{1}$ and Satoshi Hori ${ }^{5^{*}}$, \\ ${ }^{1}$ Waseda University, 169-8555 Shinjuku-ku Tokyo, Japan \\ ${ }^{2}$ Kajima Corporation, 107-8388 Minato-ku Tokyo, Japan \\ ${ }^{3}$ Showa Women's University, 154-8533 Setagaya-ku Tokyo, Japan \\ ${ }^{4}$ Shinryo Corporation, 160-8510 Chiyoda-ku Tokyo, Japan \\ ${ }^{5}$ Juntendo University, 113-8421 Bunkyo-ku Tokyo, Japan
}

\begin{abstract}
Short-distance airborne infections are often thought to occur by large droplets or direct contact. Recent studies have investigated the phenomenon of short-range airborne infection. In this study, the effect of relative humidity and short-distance exposure to cough droplet nuclei were evaluated. The evaluation model for infection risk was designed based on experimental data and previous studies and included sampling efficiency and virus survival rate. As observed in the comparison between short-range exposure and longrange exposure, the short-range exposure to the droplet nuclei by one cough was equivalent to a long-range exposure of several seconds to, depending on the number of ventilations, even for a momentary highconcentration exposure. This suggests the possibility of an infection risk corresponding to the receiving condition. Since short-range exposure and long-range exposure have different factors affecting the risk of infection, it is suggested that effective infection control should be selected for each condition. The risk of infection due to long-range exposure is considered to be sufficiently reduced to that of a single cough if the building meets the necessary number of ventilations. It was suggested that the influence of relative humidity on infection risks is smaller than other factors such as ventilation.
\end{abstract}

\section{Introduction}

We aimed to investigate the relationship between droplet nuclei concentration and relative humidity over short ranges. Simulated cough droplets were generated using a cough-generating apparatus (hereinafter cough generator), and the number of droplet nuclei that can be aspirated by a subject exposed to a cough in the immediate vicinity of the cough generator was measured. Based on experimental results, the risk of infection was evaluated and the effect of ventilation frequency, relative humidity, and distance on droplet nuclei concentration was discussed. The results of droplet nuclei concentrations at different distances are also described.

\section{Introduction Measurement of short- distance cough droplet nuclei concentration}

\subsection{Outline}

Fig 1 shows the Cough generator. We designed an experiment simulating a situation where a subject and an infected person are facing each other at a short distance. The cough generator represented a coughing infected person and the concentration of the droplet nuclei that could be inhaled by the uninfected subject was measured using an air particle sensor (ZN-PD03-S made by OMRON) at each measurement point. The experiment lasted for 11 days in total from October 2 to 5, 2017 and December 13 to 19,2017 at the Showa Women's University's artificial climate chamber (dimensions: $4.0 \mathrm{~m}$ $\times 7.9 \mathrm{~m} \times 3.0 \mathrm{~m}$ ).

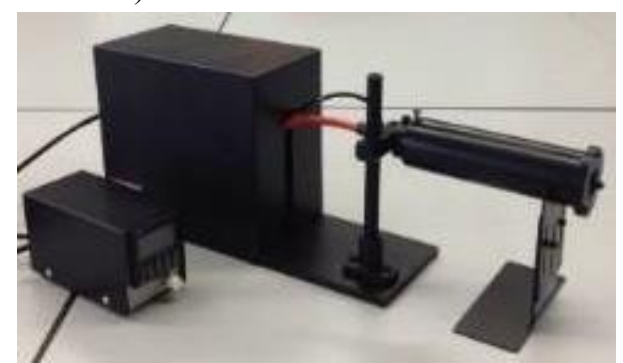

Fig 1. Cough generator

\subsection{Method}

Fig 2 shows the Measurement point plan. The sensor used in this experiment can detect and record the number of particles of 3 size categories, namely $0.3 \mu \mathrm{m}, 0.5 \mu \mathrm{m}$, and $1.0 \mu \mathrm{m}$ or more. Measurements were taken by bifurcating the suction port of the sensor and diluting with clean air.

\footnotetext{
* Corresponding author: wei@tanabe.arch.waseda.ac.jp
} 
The mouth of the cough generator was set at a height of $110 \mathrm{~cm}$ from the floor level and the measurement points were set at the same height. At each measurement point, the tip of the tube attached to the suction port was fixed upward to take measurements. Three sensors were used, and changes in droplet nuclei concentration were measured simultaneously at multiple measurement points.

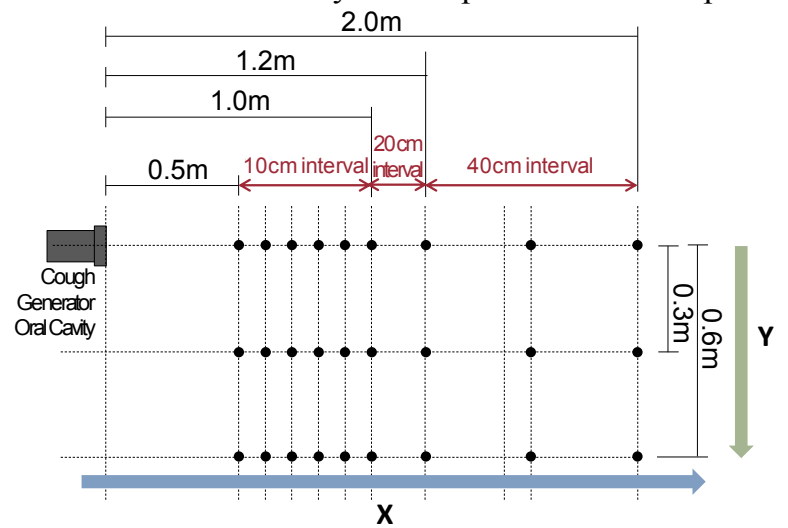

Fig 2. Measurement point plan

\subsection{Procedure}

After confirming that the environmental conditions were stable, the air conditioner was turned off to eliminate air current influence from air conditioning. The background concentration of particles was measured in the first 2 minutes and it was confirmed that the value was stable. Immediately after the background measurement, the cough generator was turned on by pressing a switch located outside the laboratory to simulate a cough, and the droplet nuclei concentration was measured for 5 minutes.

\subsection{Experimental condition}

The air temperature was set at a constant $23{ }^{\circ} \mathrm{C}$, and 2 relative humidity conditions, $30 \% \mathrm{RH}$ and $50 \% \mathrm{RH}$, were set. 27 measurement points were prepared, and droplet nuclei concentration was measured.

\subsection{Results}

\subsubsection{Effect of relative humidity}

Fig 3 shows the comparison of results of the droplet nuclei concentration and the number of droplets deposited [1]. Details on the determination of the number of deposited droplets are presented in a previous report ${ }^{1)}$. Watersensitive paper was used and the number of droplets deposited on the surface of the water-sensitive paper was recorded and the droplet trace after the simulated cough injection was analyzed.As shown in Fig 2, the number of droplet nuclei aspirated were highest at the $0.5 \mathrm{~m}$ and 0.8 $\mathrm{m}$ measurement points for the $30 \% \mathrm{RH}$ and $50 \% \mathrm{RH}$, respectively. Therefore, the relative humidity may influence the peak value distance of the droplet nuclei concentration. Splashes had $95 \%$ deposits up to $0.9 \mathrm{~m}$ in all relative humidity conditions, but it seemed like the droplet nuclei were suctioned even up to $1.6 \mathrm{~m}$. There was a high possibility of exposure to high concentrations of cough droplet nuclei up to a distance of $1.6 \mathrm{~m}$.
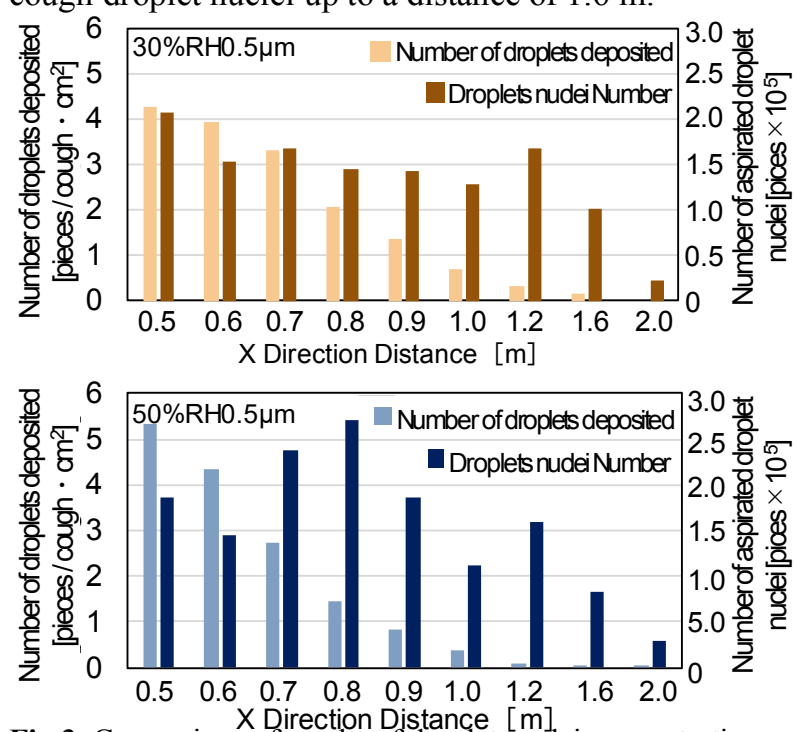

Fig 3. Comparison of results of droplet nuclei concentration and number of droplets deposited

Fig 4 shows the rate of droplet nuclei concentration ( $\mathrm{Y}=$ $0)$. In the $30 \% \mathrm{RH}$, the droplet nuclei concentration tended to increase with decrease in distance from the cough generator. In the $50 \% \mathrm{RH}$, the increase in droplet nuclei concentration was slower and the concentration tended to decrease at the $0.7 \mathrm{~m}$ and $0.8 \mathrm{~m}$ measurement points compared to the $0.5 \mathrm{~m}$ and $0.6 \mathrm{~m}$ measurement points. The rate of droplet nuclei concentration at each measurement point was affected by relative humidity increase, which also affected the speed of splashing nuclear evaporation.

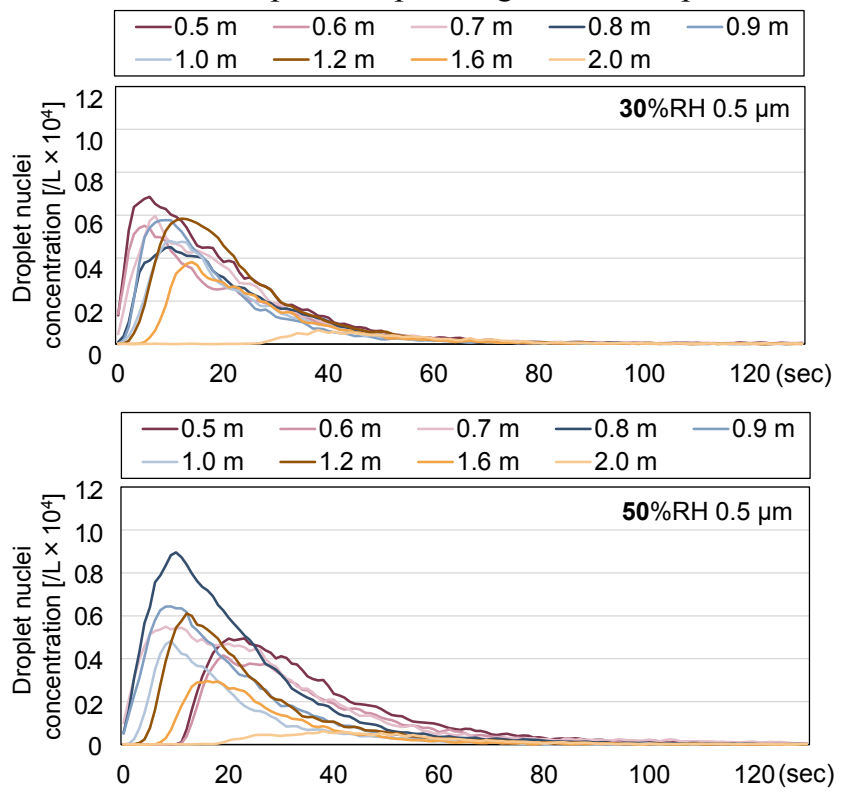

Fig 4. Temporal changes in droplet nuclei concentration $(\mathrm{Y}=0)$

\subsubsection{Effect of distance}

Fig 5 shows the integrated value of the droplet nuclei concentration at each measurement point. It was found that the peak value of the droplet nuclei concentration significantly decreased in the forward direction from the point $X=1.6 \mathrm{~m}$. The droplet nuclei also reached the lateral distance $\mathrm{Y}=0.6 \mathrm{~m}$ due to direct exposure. However, when 
compared to $\mathrm{Y}=0 \mathrm{~m}$, the number of droplet nuclei seemed to have decreased significantly. Regardless of relative humidity, the droplet nuclei concentration at $\mathrm{Y}=$ $0 \mathrm{~m}$ tended to consistently decrease at $\mathrm{X}=2.0 \mathrm{~m}$. It was observed that, in all relative humidity conditions, the peak value of the droplet nuclei concentration abruptly decreased at the $2.0 \mathrm{~m}$ measuring point.

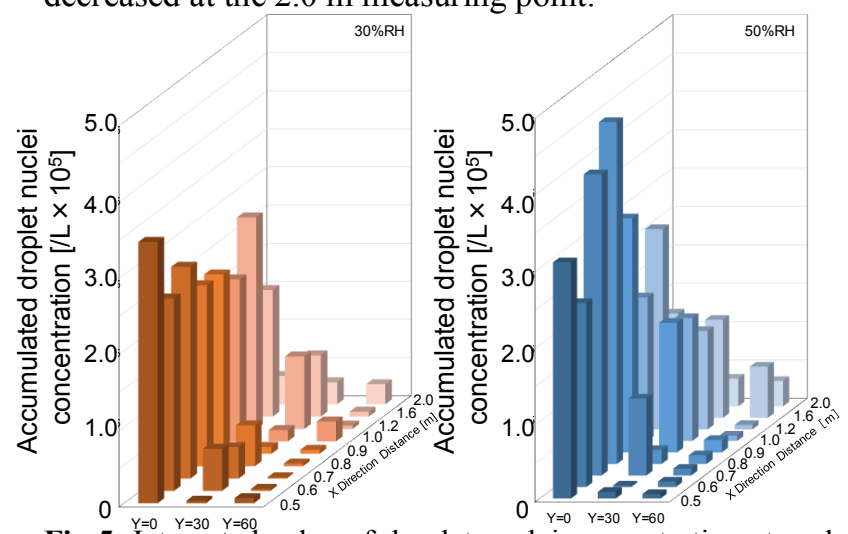

Fig 5. Integrated value of droplet nuclei concentration at each measurement point

\subsection{Experimental consideration}

In the $50 \% \mathrm{RH}$ condition, the accumulated value of the droplet nuclei concentration was highest at $\mathrm{X}=0.8 \mathrm{~m}$. It was confirmed that, in any relative humidity condition, the droplet nuclei were evenly dispersed in the $\mathrm{Y}$ direction at the $1.6 \mathrm{~m}$ measurement point or farther in the $\mathrm{X}$ direction. In other words, as the distance from the cough generator increased in the $\mathrm{X}$ direction, the droplets evaporated and the influence of relative humidity decreased. From the above findings, it can be deduced that distance has a greater influence on the relative concentration of droplet nuclei than the relative humidity condition. In addition, the relative humidity condition may affect the dispersion tendency of droplet nuclei.

\section{Discussion}

\subsection{Outline of splash nuclear infection risk evaluation formula}

We proposed a splash nuclear infection risk evaluation formula for this study based on the equation by Nicas et al. [2]. It is thought that the deposition site of the droplet nuclei is important because it affects susceptibility to infection [3]. The droplet deposition sites are categorized into 3 , namely pharynx, alveoli, and nasal region. We constructed a risk evaluation formula, designated eq (1), to determine the droplet nuclei infection probability PE.

$$
P_{E}=1-\exp \left(-\frac{I G p X t}{Q}\right)
$$

$P_{E}$ : Probability of infection

$I$ : Number of infected people

$G$ : Number of airborne bacteria released from 1 infected person per unit time

$p$ : the breathing frequency [L/hour]

$X$ : Deposition rate of infectious particles in the pharynx, alveoli, nasal region $(\alpha, \beta, \gamma)$ $t$ : Exposure time [hours]

$Q$ : Room ventilation rate [L/hour]

The number of airborne bacteria released from 1 infected person per unit time $G$ was calculated from the droplet nuclei aspiration rate based on an experiment in which $\mathrm{CO}_{2}$ gas was used as a tracer [4]. The number of droplets ejected by cough was determined at $0.5-1 \mu \mathrm{m}$. The deposition rates $\alpha, \beta, \gamma$ of infectious particles were obtained by multiplying the survival rate of the bacteria (or viruses) present in the air by the suction efficiency which changes depending on the particle size. A breathing frequency $p$ of value $600 \mathrm{~L} /$ hour was used as determined in the experiment by Liu et al. which used a breathing thermal manikin [5]. The details of the suction efficiency and survival rate of bacteria or viruses will be described later.

\subsection{Infection risk of short-distance exposure}

Fig 6 shows the infectious risk integrated value $(\mathrm{Y}=0)$. To assess the effect of relative humidity on the risk of droplet nuclei infection at each measurement point, the infection risk value of short-range exposure was integrated and each relative humidity condition was considered. As a result, in the range $\mathrm{Y}=0 \mathrm{~m}$, the effect of relative humidity varied between measurement points. However, the trend of decreasing cumulative risk of infection at the $1.6 \mathrm{~m}$ measurement point was consistent in both relative humidity conditions. It is possible that the risk of infection depends on the positional relationship with the source, rather than the relative humidity.

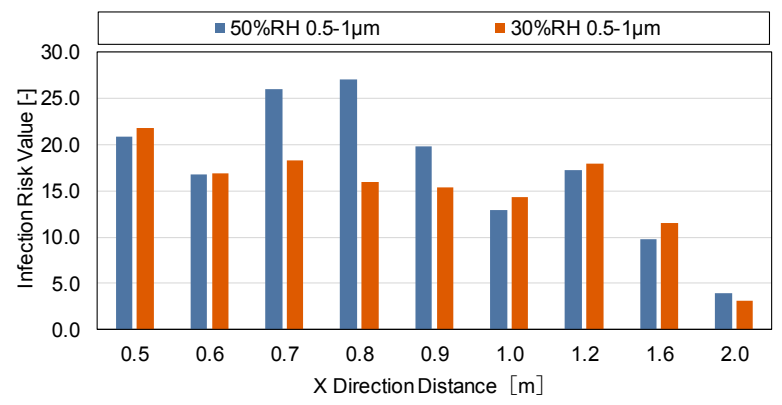

Fig 6. Infectious risk integrated value $(\mathrm{Y}=0)$

\subsection{Infection risk of long-range exposure}

In this study, we examined the effect of air change rate on the risk of infection using standard recommended 0.5, 2.0 $\mathrm{ACH}$ and 6.0 times/hour in an examination room [6]. We conducted the numerical study in a conference room of volume $42 \mathrm{~m}^{3}$ (dimensions: $3.0 \mathrm{~m} \times 5.0 \mathrm{~m} \times 2.8 \mathrm{~m}$ ). The decay rate of droplet nuclei concentration by ventilation was calculated by multiplying the initial droplet nuclei concentration of long-range exposure by the decay rate coefficient e - Qt/V based on the Seidel equation. The attenuation factor used $\mathrm{V} \mathrm{m} \mathrm{m}^{3}$ is the room volume and the initial droplet nuclei concentration for long-distance exposure at $50 \%$ RH was $308 / \mathrm{L}$. Based on the infection risk assessment of long-distance exposure, it was suggested that the risk of infection in the initial stage of long-distance exposure can be reduced by $2.0 \times 10^{4}$ relative to the non-ventilation state by performing 
ventilation. Furthermore, it was found that after 6 hours with $0.5 \mathrm{ACH}$, there was a $95 \%$ decline from the initial long-term exposure infection risk. It may therefore be possible to eliminate the risk of infection of long-term exposure by 1 cough every hour after 6 hours of ventilation. It was suggested that ventilation may have a much greater influence on the risk of infection than relative humidity.

\subsection{Infection risk control by short-range and long-distance exposures}

Fig 7 shows Temporal changes in the infection risk value. Fig 7 shows the effect of relative humidity and distance on the risk of infection with short-range exposure and the effect of ventilation on the risk of infection with long-term exposure. Short-range exposure to droplet nuclei of a single cough was observed long-term, over several seconds to several tens of minutes depending on the ventilation even for momentary high concentration exposures. This suggests that the risk may correlate with the receiving condition. Since the factors affecting the risk of infection in short-range and long-term exposures are different, effective infection control for each exposure type should be considered separately. The risk of infection from long-range exposure to a single cough is regarded as sufficiently reduced when the air change rate of the room meets the required standard. Therefore, it was posited that ventilation was more effective in infection control than relative humidity. However, in considering the influence of virus survival rate, we found that, after 6 hours, the infection risk decreased by about $4 \%$ in the $50 \% \mathrm{RH}$ and by about $1 \%$ in the $30 \% \mathrm{RH}$.

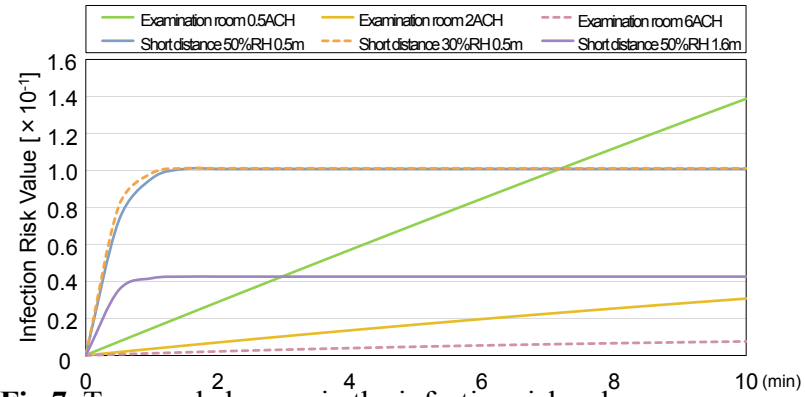

Fig 7. Temporal changes in the infection risk value

\section{Conclusion}

In this report, we report the outline and results of experiments aimed at experimentally grasping the relation between the concentration of cough and droplet nuclei and the relative humidity at short range. In addition, we evaluated the risk of droplet nuclei infection based on the experimental results and compared the risk assessment of splash nuclear infection risk in near and far distances. From the results of this experiment, we considered as follows.

1) Since the evaporation rate of droplets varies depending on the relative humidity, it is suggested that it affects the temporal change of droplet nuclei concentration. If it is within $1.6 \mathrm{~m}$ from the coughing position, possibility of short distance high concentration exposure by droplet nuclei is considered.

2) For the droplet nuclei concentration, the distance condition was more influential than the relative humidity condition. It was found that the risk of droplet nuclear infection due to short distance exposure changes more than the relative humidity depending on the positional relationship between the infected person and the uninfected person.

3) It was confirmed that the risk of droplet nuclear infection due to long-distance exposure varies greatly depending on the number of ventilation times than the relative humidity condition. Since short-range exposure and long-term exposure have different factors influencing the risk of infection, it can be said that it is necessary to implement effective infection control for each.

For future work on this topic, I would suggest including a series of very low humidity tests $(2 \%-10 \%)$ which is a typical relative humidity value met inside commercial aircraft cabins.

This research is based on the collaborative research group by Waseda University and Juntendo University. Part of this research was funded by the Young Researcher Support Project (Early Bird Program), Institute of Science and Engineering, Waseda University.

\section{References}

1. M. Ogata, M. Ichikawa, H. Tsutsumi, T. Ariga, S. Hori, S. Tanabe, Transactions of AIJ. Jounal of architecture, planning and environmental engineering, 743, 57-64, (2018)

2. M. Nicas, G. Sun, Risk Analysis, 26, 1085-1095, (2006)

3. W. Hinds, Aerosol Technology Wiley-interscience, 11, 233, (2012)

4. M. Ogata, M. Suzuki, M. Ichikawa, H. Tsutsumi, S. Tanabe, S. Hori, Architectural Institute of Japan (Kyushu), 31, 769-770, (2016)

5. Liu L, Li Y, Nielsen PV, Wei J, Jensen RL, Indoor Air, 27, 452-462, (2017)

6. Introduction to the Building Standard Law, Building Regulation in Japan

7. G. Harper, The Journal of Hygiene, 59, 479-486, (1961)

8. J. Noti, F. Blachere, C. McMillen, W. Lindsley, M. Kashon, D. Slaughter, D. Beezhold, PLOS ONE, 8, $1-8,(2013)$ 